\title{
Hawking temperature in the tunneling picture
}

\author{
Emil T. Akhmedov ${ }^{a}$, Valeria Akhmedova ${ }^{\mathrm{b}}$, Douglas Singleton ${ }^{\mathrm{b}, *}$ \\ a ITEP, B. Cheremushkinskaya, 25, Moscow 117218, Russia \\ b Physics Department, CSU Fresno, Fresno, CA 93740-8031, USA
}

Received 15 August 2006; received in revised form 21 September 2006; accepted 22 September 2006

Available online 28 September 2006

Editor: N. Glover

\begin{abstract}
We examine Hawking radiation from a Schwarzschild black hole in several reference frames using the quasi-classical tunneling picture. It is shown that when one uses, $\Gamma \propto \exp (\operatorname{Im}[\oint p d r])$, rather than, $\Gamma \propto \exp \left(2 \operatorname{Im}\left[\int p d r\right]\right)$, for the tunneling probability/decay rate one obtains twice the original Hawking temperature. The former expression for $\Gamma$ is argued to be correct since $\oint p d r$ is invariant under canonical transformations, while $\int p d r$ is not. Thus, either the tunneling methods of calculating Hawking radiation are suspect or the Hawking temperature is twice that originally calculated.
\end{abstract}

(c) 2006 Elsevier B.V. All rights reserved.

PACS: 04.62.+v; 04.70.Dy; 03.65.Xp

\section{Introduction}

The original derivation of Hawking radiation from a Schwarzschild black hole [1] was done using the methods of quantum field theory in a curved background. The physical picture given for this process was in terms of particles tunneling through the horizon which was not directly connectable with the field theory derivation. Relatively recently this physical picture has been given support by quasi-classical tunneling calculations [2-7]. The present approach, where we use the HamiltonJacobi equations to study black hole radiation, is that used in $[4,6]$. All the above works are more directly connected to the physical picture of Hawking radiation as tunneling, and are more transparent and less complex than the field theory derivation. In a recent work [8] we studied Hawking-like radiation in various gravitation backgrounds using the quasi-classical tunneling approach and the Hamilton-Jacobi equations. In the case of Schwarzschild black holes we found the physically questionable result that the Hawking temperature apparently depended on the type of coordinates used (e.g. Schwarzschild, Painlevé,

\footnotetext{
* Corresponding author.

E-mail addresses: akhmedov@itep.ru (E.T. Akhmedov), lera@itep.ru (V. Akhmedova),dougs@csufresno.edu (D. Singleton).
}

isotropic). Further in [9] it was argued that since the quantity $\int p d r$ is not invariant under canonical transformations, the tunneling probability or decay rate, $\Gamma \propto \exp \left(2 \operatorname{Im}\left[\int p d r\right]\right)$, is not a proper observable in this particular case of tunneling through the black hole horizon. In this Letter we will discuss these issues and suggest resolutions to these problems with the tunneling calculations of Hawking radiation. We give arguments that if the tunneling calculations are correct then the temperature of the Hawking radiation should be twice as large as originally calculated.

\section{Hamilton-Jacobi equation approach to black hole radiation via tunneling}

To study the thermal radiation given off in some gravitational background we consider the Klein-Gordon equation in a curved background:

$\left[-\frac{\hbar^{2}}{\sqrt{-g}} \partial_{\mu} g^{\mu \nu} \sqrt{-g} \partial_{\nu}+m^{2}\right] \phi=0$.

The signature of the metric is $(-1,1,1,1)$ and $d s^{2}=g_{\mu v}(x) \times$ $d x^{\mu} d x^{\nu}, g_{\mu \nu} g^{\nu \alpha}=\delta_{\mu}^{\alpha}$. In this Letter we do not take into account the back-reaction of gravity on the quantum fluctuations of the scalar field. 
We are looking for the solutions of (1) having the form: $\phi(x) \propto \exp \left\{-\frac{i}{\hbar} S(x)+\cdots\right\}$. Inserting this into (1) and taking the limit $\hbar \rightarrow 0$ we find to order $\hbar^{0}$ the following equation

$g^{\mu v} \partial_{\mu} S \partial_{\nu} S+m^{2}=0$,

which is the relativistic Hamiltonian-Jacobi equation for the classical action of a relativistic particle in a curved background. The condition under which our approximation is valid is worked out in [10].

Since the Schwarzschild metric is stationary it has a timelike Killing vectors. Thus, we will look for particle-like solutions of (2) which behave as $S=E t+S_{0}(\vec{x})$, where $x_{\mu}=(t, \vec{x})$ and $E$ is the energy of the particle. The wave function for such solutions behaves as $\phi \propto e^{-\frac{i}{\hbar} E t}$ and corresponds to a state with definite energy. These are the states which are observed by detectors.

If the solution $S_{0}(\vec{x})$ of (2) has a non-zero imaginary part for some particle trajectory, this implies that the gravitational background in question is unstable with respect to radiation of such definite energy states. In this case the wave function behaves as: $\phi \propto e^{-\frac{1}{\hbar} \operatorname{Im} S_{0}}$, which describes tunneling of the particle through the gravitational barrier. This leads to the decay of the background with the rate given by $\Gamma \propto|\phi|^{2} \propto e^{-\frac{2}{\hbar} \operatorname{Im} S_{0}}$.

In Schwarzschild coordinates an uncharged, non-rotating black hole with mass $M$ has a metric of the form

$d s^{2}=-\left(1-\frac{2 M}{r}\right) d t^{2}+\frac{d r^{2}}{\left(1-\frac{2 M}{r}\right)}+r^{2} d \Omega^{2}$.

For this metric and for radial trajectories which are independent of $\theta, \varphi$ the Hamilton-Jacobi equation becomes

$-\frac{1}{\left(1-\frac{2 M}{r}\right)}\left(\frac{\partial S}{\partial t}\right)^{2}+\left(1-\frac{2 M}{r}\right)\left(\frac{\partial S}{\partial r}\right)^{2}+m^{2}=0$.

For the definite energy state we obtain

$$
-\frac{E^{2}}{\left(1-\frac{2 M}{r}\right)}+\left(1-\frac{2 M}{r}\right)\left(\frac{d S_{0}}{d r}\right)^{2}+m^{2}=0 .
$$

Despite the fact that the Schwarzschild metric has two disjoint parts separated by $r=2 M$, we can nevertheless consider solutions of (5) in these two regions and glue them by going around the pole in the complex $r$-plane. The solution is

$S_{0}= \pm \int_{0}^{+\infty} \frac{d r}{\left(1-\frac{2 M}{r}\right)} \sqrt{E^{2}-m^{2}\left(1-\frac{2 M}{r}\right)}$,

where the limits of integration are chosen such that the particle goes through the horizon $r=2 M$. We focus on the integration through $r=2 M$ since this is exactly where the complex part of $S_{0}$ comes from. The $+(-)$ sign in front of this integral indicates that the particle is ingoing (outgoing). Thus both incoming and outgoing particles face barriers as should be expected for a barrier penetration/tunneling problem. However classically this is odd since one expects a classical particle to face a barrier only when it is outgoing not incoming. This point will be discussed in detail later, but note that for virtual particle pairs the positive energy component must tunnel out from the horizon, while the negative energy component must tunnel in through the horizon.

Because there is a pole at $r=2 M$ along the path of integration the integral will just be the Cauchy principle value. The imaginary part of the principle value of (6) is given by the contour integral over a small half-loop going around the pole from below from left to right. This choice of the contour seems to correspond to the small "trajectory" of the particle just crossing the horizon. It is worth mentioning at this point that there is no real trajectory of a classical particle corresponding to this contour even in Euclidian time. This observation makes the whole quasi-classical approach questionable. However, in a moment we will give independent arguments why this is nevertheless correct.

First, let us explicitly take the imaginary part of the principle value. We make the change of variables $r-2 M=\epsilon e^{\mathrm{i} \theta}$. Then

$$
\begin{aligned}
\operatorname{Im} S_{0}= \pm \lim _{\epsilon \rightarrow 0} \int_{\pi}^{2 \pi} \frac{\left(2 M+\epsilon e^{\mathrm{i} \theta}\right) \epsilon e^{\mathrm{i} \theta} \mathrm{i} d \theta}{\epsilon e^{\mathrm{i} \theta}} \\
\quad \times \sqrt{E^{2}-m^{2}\left(1-\frac{2 M}{2 M+\epsilon e^{\mathrm{i} \theta}}\right)} \\
= \pm 2 \pi M E .
\end{aligned}
$$

Using this result for $\operatorname{Im} S_{0}$ for the outgoing particle the decay rate of the black hole is $\Gamma \propto e^{\frac{-4 \pi M E}{\hbar}}$. From the above expression for $\Gamma$ one sees that it is just the Boltzmann weight with the temperature $T=\hbar / 4 \pi M$.

Before continuing our quasi-classical analysis in other frames a comment on the form of the decay rate is in order. The behavior of the decay rate, $\Gamma \propto e^{-\frac{4 \pi M E}{\hbar}}$, seems strange from the point of view of quasi-classics. The tunneling rate decreases with increasing energy, which is contrary to the "quasi-classical" intuition. However, there is an obvious explanation for this behavior: the greater the energy of a particle the stronger it is attracted to the gravitating body (black hole), hence, the harder it is for it to escape.

Now let us come back to the imaginary part of the action. We have obtained the temperature $T=\hbar / 4 \pi M$ which is twice the temperature originally calculated by Hawking. We now look further into this discrepancy. In [3] the tunneling calculation was performed not in the Schwarzschild frame of (3) but in the Painlevé frame

$d s^{2}=-\left(1-\frac{2 M}{r}\right) d t^{2}+2 \sqrt{\frac{2 M}{r}} d r d t+d r^{2}+r^{2} d \Omega^{2}$.

(The use of the Painlevé frame to study tunneling in thin film ${ }^{3} \mathrm{He}$ black hole analog systems was first employed in [2].) The Painlevé frame is obtained from the Schwarzschild frame by making the following transformation of the time coordinate

$d t^{\prime}=d t+\frac{\sqrt{\frac{2 M}{r}} d r}{1-\frac{2 M}{r}}, \quad r^{\prime}=r, \quad \Omega^{\prime}=\Omega$.

This metric is regular (i.e. does not have the horizon for the incoming particles) at $r=2 M$. However the notion of time is 
changed with respect to the Schwarzschild coordinates, so that the Hamilton-Jacobi equation for the definite energy state becomes

$-E^{2}+\left(1-\frac{2 M}{r}\right)\left(\frac{d S_{0}}{d r}\right)^{2}+2 \sqrt{\frac{2 M}{r}} E \frac{d S_{0}}{d r}+m^{2}=0$.

The solution of this equation is

$$
\begin{aligned}
S_{0}= & -\int_{C} \frac{d r}{1-\frac{2 M}{r}} \sqrt{\frac{2 M}{r}} E \\
& \pm \int_{C} \frac{d r}{1-\frac{2 M}{r}} \sqrt{E^{2}-m^{2}\left(1-\frac{2 M}{r}\right)} .
\end{aligned}
$$

This result cannot be obtained from (6) via a change of integration variables because the transformation (9) does affect the time-like Killing vector. One can see that (11) differs from (6) by the first term. The first term in (11) arises from the coordinate change in (9), since

$\int E d t+S_{0}=\int E d t^{\prime}-\int \frac{d r}{1-\frac{2 M}{r}} \sqrt{\frac{2 M}{r}} E+S_{0}$,

where $S_{0}$ is given by (6). Physically this new coordinate system corresponds to $r$-dependent, singular shift of the initial time.

If we choose the plus sign in (11) (which corresponds to an incoming particle) and the contour $C$ as in (7), we find $\operatorname{Im} S_{0}=0$ since the first and second terms in (11) have the same magnitude. Thus incoming particles do not see a barrier or horizon. If on the other hand we choose the minus sign in (11) and the contour $C$ as before the result is

$\operatorname{Im} S_{0}=-4 \pi M E$.

This is twice the result of (7) because the first integral in (11) gives the same contribution to the complex part of $S_{0}$ as the second one. Thus in this frame, if one uses $\Gamma \propto|\phi|^{2} \propto e^{-\frac{2}{\hbar} \operatorname{Im} S_{0}}$ for the decay rate, one apparently recovers the original temperature of the black hole as calculated in [1] namely $T=\hbar / 8 \pi M$. Note that in Hawking's original derivation he also did not use the Schwarzschild frame but rather used a frame where the time $t^{\prime}$ was related to the Schwarzschild time via $d t^{\prime}=$ $d t+d r /\left(1-\frac{2 M}{r}\right)$. As in the transformation from Schwarzschild frame to Painlevé frame, this involves a shifting of the time coordinate.

In [6] it was argued that this apparent disagreement between the results in the Schwarzschild frame and in the Painlevé frame (or in the frame used by Hawking in [1] mentioned in the previous sentence) was a result of the bad behavior of the Schwarzschild coordinates at $r=2 M$. By making a change of spatial variables to a frame where the coordinates were better behaved at the horizon it was claimed one would recover the original result of Hawking for the temperature. As a particular example one could consider isotropic coordinates which are obtained from the Schwarzschild coordinates via the change of variables [6]

$r=\rho\left(1+\frac{M}{2 \rho}\right)^{2}$.
With this the Schwarzschild metric (3) becomes

$d s^{2}=-\left(\frac{\rho-\frac{M}{2}}{\rho+\frac{M}{2}}\right)^{2} d t^{2}+\left(\frac{\rho+\frac{M}{2}}{\rho}\right)^{4}\left(d \rho^{2}+\rho^{2} d \Omega^{2}\right)$.

Now instead of (6) we find

$S_{0}= \pm \int \frac{\left(\rho+\frac{M}{2}\right)^{3}}{\left(\rho-\frac{M}{2}\right) \rho^{2}} \sqrt{E^{2}-m^{2}\left(\frac{\rho-\frac{M}{2}}{\rho+\frac{M}{2}}\right)^{2}} d \rho$.

If one does the contour integration in the same manner as in (7) by making a semi-circular contour one apparently finds that $\operatorname{Im} S_{0}= \pm 4 \pi M E$ [6]. However there is a subtle point: one must also deform the contour from (7) using (14) and when this is done the semi-circular contour of (7) gets transformed into a quarter circle so that one gets $\mathrm{i} \frac{\pi}{2}$ Residue $^{1}$ rather than $\mathrm{i} \pi$ Residue. One could already guess this because from (14) $\rho \simeq \sqrt{r}$ which for the contour in (7) means the semi-circle contour becomes a quarter circle. In detail

$$
\begin{aligned}
r= & 2 M-\epsilon e^{\mathrm{i} \theta} \\
= & \rho+M+\frac{M^{2}}{4 \rho} \\
& \rightarrow \rho=\frac{1}{2}\left(M+\epsilon e^{\mathrm{i} \theta} \pm\left(2 M+\epsilon e^{\mathrm{i} \theta}\right) \sqrt{\epsilon} e^{\mathrm{i} \theta / 2}\right) .
\end{aligned}
$$

The leading order in epsilon is now $\sqrt{\epsilon}$ so in the limit $\epsilon \rightarrow 0$ we find from the above equation $\rho-\frac{M}{2}=M \sqrt{\epsilon} e^{\mathrm{i} \theta / 2}$ instead of $r-2 M=\epsilon e^{\mathrm{i} \theta}$. Since $e^{\mathrm{i} \theta}$ becomes $e^{\mathrm{i} \theta / 2}$, one sees that the semicircle contour of the Schwarzschild frame gets transformed into a quarter circle in the isotropic coordinate frame so that the result of integrating (16) is $\mathrm{i} \frac{\pi}{2}$ Residue and we find again $\operatorname{Im} S_{0}= \pm 2 \pi M E$.

In [6] general arguments are given that one should work with the proper spatial distance as defined by

$d \sigma^{2}=\frac{d r^{2}}{B(r)}+r^{2} d \Omega^{2} \rightarrow \sigma=\int \frac{d r}{\sqrt{B(r)}}$,

where $B(r)=1-\frac{2 M}{r}$. In the last step we are considering only the radial part or the s-wave contribution to the tunneling. In [6] by considering the near horizon approximation (i.e. $\left.B(r)=B^{\prime}(r=2 M)(r-2 M)+\cdots\right)$ one finds

$\sigma=2 \sqrt{2 M} \sqrt{r-2 M}+\cdots$.

From this one sees that in general the contour from (7) defined via $r-2 M=\epsilon e^{\mathrm{i} \theta}$ will always be transformed from a semicircle to a quarter circle because of the square root in (19). Thus any coordinate transformation of (7) involving only spatial coordinates will yield the same result for the temperature as (7). This can also be seen from the point that making a coordinate transformation involving the spatial coordinates is just a change of integration variables and should not change the result.

Thus, if one uses Schwarzschild coordinates or any coordinates related to them via a transformation of spatial coordinates

\footnotetext{
1 It is this choice of the contour, rather than any other one, which corresponds to going from inside to outside the horizon.
} 
one gets twice the original Hawking temperature, while transformations involving the time coordinate appear to give the original Hawking result. How can one reconcile these various results? In fact, a detector will only measure one or the other of these temperatures via say the rate of flipped and un-flipped spins.

We now show that even in the Painlevé frame one obtains twice the Hawking temperature if one takes the proper exponent in the expression for $\Gamma$. In [9] the tunneling approach to Hawking radiation was criticized based on the fact that $2 \operatorname{Im} S_{0}=2 \operatorname{Im} \int p d r$ is not invariant under canonical transformations and thus $\Gamma \propto \exp \left(2 \operatorname{Im} S_{0}\right)=\exp \left(2 \operatorname{Im}\left[\int p d r\right]\right)$ is not a proper observable; one could change $\Gamma$ by making a canonical transformation. In [9] it is argued that since the closed contour integral, $\oint p d r$, is invariant under canonical transformations one should take for the decay rate $\Gamma \propto \exp (\operatorname{Im}[\oint p d r])$. The relationship between the two expressions can be seen by considering a closed path that goes from $r=r_{\mathrm{i}}$, which in just inside the horizon, to $r=r_{\mathrm{o}}$ just outside the horizon

$$
\oint p d r=\int_{r_{\mathrm{i}}}^{r_{\mathrm{o}}} p_{\text {out }} d r+\int_{r_{\mathrm{o}}}^{r_{\mathrm{i}}} p_{\text {in }} d r .
$$

The points, $r_{\mathrm{i}}, r_{\mathrm{O}}$ are chosen to straddle the horizon since this is where the imaginary part of $S_{0}$ comes from. If, as suggested in [9], one takes (20) to define the exponent in $\Gamma$ then one finds that all three frames considered-Schwarzschild, Painlevé and isotropic-yield the same result namely $\operatorname{Im} \oint p d r=4 \pi M E$ which then in all cases gives $\Gamma \propto e^{\frac{-4 \pi M E}{\hbar}}$ and a temperature of, $T=\hbar / 4 \pi M$, twice the Hawking temperature. In the Schwarzschild and isotropic coordinates the $p_{\text {out }}$ and $p_{\text {in }}$ have equal magnitude, but opposite signs - the $+(-)$ signs correspond to $p_{\text {out }}\left(p_{\text {in }}\right)$ in (6), (16). For these two coordinate frames one can see why $\oint p d r=2 \int_{r_{\mathrm{i}}}^{r_{\mathrm{o}}} p_{\text {out }} d r=2 \int_{r_{\mathrm{o}}}^{r_{\mathrm{i}}} p_{\text {in }} d r$. In the Painlevé coordinates the entire contribution comes from $p_{\text {out }}$, since for $p_{\text {in }}$ the two contributions in (11) cancel. For the Painlevé coordinates $\oint p d r \neq 2 \int_{r_{\mathrm{i}}}^{r_{\mathrm{o}}} p_{\text {out }} d r \neq 2 \int_{r_{\mathrm{o}}}^{r_{\mathrm{i}}} p_{\text {in }} d r$. The fact that one gets a contribution from both incoming and outgoing particles when one uses Schwarzschild or isotropic coordinates is in accordance with the idea that in a proper tunneling problem one should face a barrier regardless of whether one moves from left (inside) to right (outside) or from right (outside) to left (inside) across the barrier. However from the classical point of view this appears odd since a classical particle can easily cross the horizon going inward. It is only crossing the horizon in the outward direction that is forbidden for a classical particle. However in the tunneling picture one is looking at virtual pairs of negative-positive energy particles which are fluctuating out of the vacuum near the horizon. For such a virtual pair just inside the horizon, the positive energy part must tunnel out of the horizon with the negative energy component going inward. Just outside the horizon it is the negative energy component which must tunnel through the horizon with the positive energy component going outward. Thus contrary to classical intuition the horizon represents a two way barrier when one considers virtual particle pairs.
We give two further arguments supporting our conclusions. The calculation of the Unruh temperature in our previous paper [8] is in accordance with the considerations of the previous paragraph. Similar to the Schwarzschild metric, the Rindler metric has a horizon for the particles going both ways through its singular point. Also, the corresponding decay rate is given by $\log \Gamma \propto \operatorname{Im} \oint p d r$, which gives the correct Unruh temperature $T=a / 2 \pi$, where $a$ is the acceleration. The second argument follows by considering the scattering problem in the black hole background (see e.g. [11]). The Klein-Gordon equation in the Schwarzschild background:

$$
\begin{aligned}
& {\left[-\frac{1}{\left(1-\frac{2 M}{r}\right)} \frac{\partial^{2}}{\partial t^{2}}+\frac{1}{r^{2}} \frac{\partial}{\partial r} r^{2}\left(1-\frac{2 M}{r}\right) \frac{\partial}{\partial r}\right.} \\
& \left.+\frac{1}{r^{2}} \Delta(\theta, \varphi)-m^{2}\right] \phi=0,
\end{aligned}
$$

where $\Delta(\theta, \varphi)$ is the angular part of the Laplacian. We would like to find the behavior of the constant energy solution $(\phi=$ $\left.e^{\mathrm{i} E t} \phi_{E}\right)$ in the vicinity of the horizon $r=2 M$. Changing the variables to $z=r-2 M$, the Klein-Gordon equation approximately reduces to:

$\left[(2 M E)^{2}+z \frac{d}{d z} z \frac{d}{d z}\right] \phi_{E}=0$.

Thus, near the horizon the wave function behaves as follows:

$\phi_{E} \propto z^{\mathrm{i} 2 M E} \propto(r-2 M)^{\mathrm{i} 2 M E}$.

The power in this formula is directly related to the imaginary part of the action which we considered above. To solve the scattering problem in question we have to connect solutions inside and outside the horizon [11]. This is achieved by going to the complex $r$ plane and taking the above mentioned contour around $r=2 M$. In this way we obtain a damping factor of $e^{-2 \pi M E}$ [11] which corresponds to a temperature of $T=\hbar / 4 \pi M$.

As a final comment tunneling calculations in general (if one has canonical invariance) seem to give a factor of two greater temperature as compared quantum field theory calculations. In [12] the tunneling calculation of the Gibbons-Hawking temperature of a de Sitter spacetime gave a temperature twice that of the quantum field theory calculation, but their explanation of the discrepancy in question is not applicable to our case. The Unruh effect is only case we have examined thus far where the tunneling and other calculations agree.

\section{Conclusions}

We have shown that by requiring canonical invariance (i.e. using quantity $\oint p d r$ to define the exponent of the decay rate, $\Gamma$ ) the tunneling calculations of black hole radiation all give a temperature that is twice that was originally calculated by Hawking. There are two possible conclusions: (i) the tunneling calculations are not correct in detail or (ii) the Hawking temperature is twice as large as the original calculations suggest. (A third possibility has been suggested to us [13]: that 
canonical invariance is not a requirement in the tunneling calculations as applied to black holes. We can find no argument why this should be and thus do not take this option into account.) We now discuss the pros and cons of these two possibilities. If one takes the second possibility as correct then the tunneling calculations, although corresponding to the physical picture of Hawking radiation as tunneling through the horizon, are only a heuristic guide which in detailed calculations do not give the correct numerical factor for the temperature. However, the standard calculation of the Hawking temperature is not airtight; there are open questions: the appearance of trans-Planckian energies at intermediate stages of the calculation and the unknown effect of quantum gravitational corrections [14,15]. Ref. [16] gives an excellent discussion of these issues and other open issues in the standard derivation of Hawking radiation. Finally we note that in the original calculation of Hawking radiation, and in almost all subsequent calculations, the Schwarzschild frame is not used, but rather the frame is used where the time $t^{\prime}$ is related to the Schwarzschild time via $d t^{\prime}=d t+d r /\left(1-\frac{2 M}{r}\right)$. This is similar to the transformation (9) that takes one from Schwarzschild to Painlevé coordinates. Changing the time coordinate in this way changes the quantization of the fields, and could lead to subtleties such as those that arose when one did the tunneling calculation in Painlevé coordinates. In fact, in different frames (Schwarzschild or isotropic) even using Hawking's approach one should use a different basis of Fourier harmonics, which could then lead to different results for the black hole temperature. There was a previous suggestion that the temperature of Hawking radiation should be twice the originally calculated value [17]. In [17] it was argued that any horizon splits the Universe into two parts, both of which give a contribution to the thermal radiation. This is similar to the tunneling picture we sketched at the end of the last section: virtual particles just inside the horizon and just outside the horizon, both contribute to the tunneling and thus to the thermal radiation.

We close by making some observations on the effects of having a Hawking temperature twice as large as originally calculated. Astrophysically doubling the Hawking temperature would have no experimental significance, since for astronomical black holes Hawking radiation is too weak to detect. However, in some extra-dimensional models, gravity becomes strongly coupled at the $\mathrm{TeV}$ scale with the possibility of creating micro black holes at the LHC [18]. These micro black holes would rapidly decay via Hawking radiation and in this case the factor of two difference would be noticeable. Another area where having the Hawking temperature twice as large would make a difference is in the entropy of a black holes. For a non-rotating, chargeless, black hole the 1st law of black hole thermodynamics reads (taking $G=1, c=1, k_{B}=1$ )

$d M=\frac{\kappa}{8 \pi} d A \quad$ with $\kappa=\frac{1}{4 M}$.

Comparing with the ordinary 1st law $(d U=T d S)$ and assuming that the Hawking temperature is $T=\hbar / 8 \pi M$ then leads to the Hawking-Bekenstein [19] expression for black hole en- tropy: $d S=d A / 4 \hbar \rightarrow S=A / 4 \hbar$ where $A$ is the area of the horizon. One of the theoretical tests of quantum gravity theories such as string theory or loop quantum gravity is to calculate the entropy of a black hole by counting the microscopic states. In certain special situations (i.e. extremal, supersymmetric black holes) there have been string theory calculations [20] of black hole entropy. A review of this topic can be found in [21]. In loop quantum gravity there have also been calculations of black hole entropy [22]. The goal is to reproduce the result $S=A / 4 \hbar$. However if as indicated by the tunneling calculations the temperature is really $T=\hbar / 4 \pi M$ this would imply $S=A / 8 \hbar$ which would be important for quantum gravity calculations which try to reproduce the black hole entropy by counting microscopic states.

\section{Acknowledgements}

A.E.T. would like to thank S. Dubovski, S. Sibiryakov, V. Rubakov and especially A. Morozov and J. Bjorken for illuminating discussions. This work supported by the following grants: RFBR 04-02-16880 and the Grant from the President of Russian Federation for support of scientific schools, and a CSU Fresno International Activities Grant.

\section{References}

[1] S.W. Hawking, Commun. Math. Phys. 43 (1975) 199.

[2] G.E. Volovik, JETP Lett. 69 (1999) 662.

[3] M.K. Parikh, F. Wilczek, Phys. Rev. Lett. 85 (2000) 5042; M.K. Parikh, Int. J. Mod. Phys. D 13 (2004) 2351.

[4] K. Srinivasan, T. Padmanabhan, Phys. Rev. D 60 (1999) 24007; S. Shankaranarayanan, T. Padmanabhan, K. Srinivasan, Class. Quantum Grav. 19 (2002) 2671.

[5] A.J.M. Medved, E.C. Vagenas, Mod. Phys. Lett. A 20 (2005) 1723; A.J.M. Medved, E.C. Vagenas, Mod. Phys. Lett. A 20 (2005) 2449; M. Arzano, A.J.M. Medved, E.C. Vagenas, JHEP 0509 (2005) 037; E.C. Vagenas, Nuovo Cimento B 117 (2002) 899.

[6] M. Angheben, M. Nadalini, L. Vanzo, S. Zerbini, JHEP 0505 (2005) 014; M. Nadalini, L. Vanzo, S. Zerbini, J. Phys. A 39 (2006) 6601.

[7] R. Kerner, R.B. Mann, Phys. Rev. D 73 (2006) 104010.

[8] E. Akhmedov, et al., hep-th/0605137.

[9] B.D. Chowdhury, hep-th/0605197.

[10] L. Landau, L. Lifshitz, Quantum Mechanics (Non-Relativistic Theory), third ed., Elsevier, Amsterdam, 1977, Chapter 7.

[11] R. Brout, S. Massar, R. Parentani, Ph. Spindel, Phys. Rep. 260 (1995) 329.

[12] R. Brout, Ph. Spindel, Nucl. Phys. B 348 (1991) 405.

[13] M.K. Parikh, private communication.

[14] G. Gibbons, in: R. Ruffini (Ed.), Proceedings of the first Marcel Grossman Meeting on General Relativity, 1997, p. 449.

[15] T. Jacobson, Phys. Rev. D 44 (1991) 1731.

[16] A. Helfer, Rep. Prog. Phys. 66 (2003) 943.

[17] G. 't Hooft, J. Geom. Phys. 1 (1984) 45.

[18] T. Banks, W. Fischler, hep-th/9906038;

S. Dimopoulos, G. Landsberg, Phys. Rev. Lett. 87 (2001) 161602;

S.B. Giddings, S. Thomas, Phys. Rev. D 65 (2002) 056010.

[19] J. Bekenstein, Phys. Rev. D 7 (1973) 2333.

[20] A. Strominger, C. Vafa, Phys. Lett. B 379 (1996) 99.

[21] E. Akhmedov, Int. J. Mod. Phys. A 15 (2000) 1.

[22] A. Ashtekar, et al., Phys. Rev. Lett. 80 (1998) 904. 